\title{
Research on Regulation of Receptor Signalling Pathway of HeLa Cells by Clock Molecule DEC1
}

\author{
Weiya Lang ${ }^{1}$, Yu jing ${ }^{1}$, Hongguang $\mathrm{Bao}^{2}$, Yongde Hong ${ }^{1}$, He \\ lan ${ }^{1},{ }^{*}$ Hongming Pan ${ }^{1}$ \\ ${ }^{1}$ Qiqihar Medical University, Qiqihar, China, 161000, ${ }^{2}$ The Second \\ Affiliated Hospital of Qiqihar Medical University, Qiqihar, China, 161000 \\ *Corresponding Author: Hongming Pan
}

\begin{abstract}
Objective: To make a study of the key factors from the Src to the nuclear transcription factor ERK signaling pathway as the target and to explore the role of DEC1 mediated ERK signaling pathway in cervical cancer and its mechanism. Methods: On the basis of screening DEC1 positive cells, the Hela cells with the highest positive rate of FRtt and the lowest DEC1 positive rate of MCF-7 cells were used as the experimental objects, and the growth of cervical cancer cells was detected by cell counting. Results: Src ERKl and C Hela cells, Jun mRNA relative expression was significantly lower than that in MCF-7 cells, the differences were statistically significant $(\mathrm{P}<0.05)$ : Hela and MCF-7 cells ERK2 and $\mathrm{C}$ - Fos mRNA relative expression was no significant difference $(\mathrm{P}>0.05)$. Conclusion: DEC1 can down regulate the expression of ERK upstream factor Src and protein in Hela cells of cervical cancer cell line mRNA, suggesting that DEC1 has other intermediate factors in the activation of ERK signaling pathway. Keywords: clock molecule, cervical cancer cell, HeLa cells, signalling pathway
\end{abstract}

\section{Introduction}

Cervical cancer is one of the main causes of death among women, and the incidence rate of cervical cancer is the highest among all female gynaecological tumours in second place. There are about 13.2 million new cases of cervical 
cancer in China each year [1], accounting for $28 \%$ of the total number of new cases of cervical cancer. Cervical cancer is a serious public health problem that threatens the health of women. Effectively solve the problem of women's two cancer prevention is imminent.

The occurrence and development of cervical cancer is a complex pathological process, it is clear that human papillomavirus (human papillomavirus, HPV) infection, especially HPV16, 18 high-risk HPV (HR - HPV) persistent infection is a major cause of cervical cancer, but not the only factor. When the cell is stimulated by the external signal, the signal can be identified, amplified and transmitted through a series of signal pathways in the cell, which eventually leads to a series of physiological and biochemical changes. Extracellular signal regulated kinase (ERK) is a mitogen activated protein kinase, one of the members of the family, can be activated by many stimuli, promote cell growth and proliferation. ERK can be activated by upstream Ras or Sic, which is an active form of phosphorylation, and it is generally believed that the phosphorylation of ERK represents the activation of the Ras / Raf / MAPK pathway. After the activation of most ERK translocation to the nucleus, the phosphorylation of a series of nuclear transcription factor, expression of these proteins involved in the regulation of cell growth, development, differentiation, apoptosis and other physiological processes. Therefore, the relationship between ERK signal transduction pathway and tumour is attracting more and more attention. The results showed that ERK activity was detected in most tumour cells. The mechanism of ERK phosphorylation induced by DEC1 in cervical cancer and the effect of downstream will need to be further studied [2].

\section{Materials and Methods}

\subsection{Materials}

(1) DMEM complete medium $(100 \mathrm{~m} 1): 10 \%$ fetal bovine serum, 1\%10U / LAL mycillin double antibody solution, namely with medium volume $100 \mathrm{ml}$, type DMEM sealing. (2) MEM.EBSS complete medium (100m1):10\% fetal bovine serum, penicillin and streptomycin resistant double solution, solution volume $100 \mathrm{ml}$, sealed with the use of MEM - EBSS $46 \mathrm{C}$ culture preservation. (3) MEM - EBSS (NEAA) complete medium (100m1):10\% fetal bovine serum, penicillin and streptomycin resistant double solution, 1\%NEAA, liquid volume $100 \mathrm{ml}$, sealed with the use of MEM - EBSS training, 4 degrees to save. (3) SDS $10 \%$ SDS: $109,50 . \mathrm{C}$ bath solution in $80 \mathrm{ml}$ deionized water to be dissolved after constant volume to $100 \mathrm{M} 1$ at room temperature. (4) $1.5 \mathrm{~mol} /$ LTris (pH8.8) 8.179 Tris $100 \mathrm{ml}$ in the bottle, add $80 \mathrm{ml}$ deionized water to stir to dissolve, $\mathrm{pH}$ value to 8.8 (concentrated hydrochloric acid), set to $100 \mathrm{ml}$, high temperature and high pressure sterilization, preservation of 4 6C. (5) $1.0 \mathrm{~mol} / \mathrm{L}$ Tris (pH6.81): take $12.11 \mathrm{~g}$ Tris, dissolved in $80 \mathrm{ml}$ deionized water, with concentrated hydrochloric acid to adjust PH to 6.8 , the capacity of $100 \mathrm{ml}$, and high pressure 30min. (6) Tris electrophoresis buffer preparation: 15.1 ore glycine 449+SDS5.09 into a $1 \mathrm{~L}$ beaker, $800 \mathrm{ml}$ dissolved in deionized water, constant volume to $1000 \mathrm{ml}$, 
mixed evenly at room temperature, diluted 5 times with. (7) SDS - PAGE glue (separation gel): ultra-pure water $2.3 \mathrm{ml}$, acrylamide $5.0 \mathrm{ml}, 1.5 \mathrm{~mol} / \mathrm{L} \mathrm{Hcl}(\mathrm{PH}=8.8)$ $2.5 \mathrm{ml}, 10 \%$ SDS O.1ml, 10\%AP 0.1ml, TEMED 0.004ml, Tris.

\subsection{Methods}

Hela, MCF-7, MDA-MB-231 and CaSki four kinds of cancer cells were cultured, four cell lines were derived from the China Academy of Medical Sciences Institute of basic cell resource center. (1) cell culture: Hela, MCF-7, MDA-MB231 and CaSki cells respectively with DMEM medium (10\% fetal bovine serum $+100 \mathrm{u} / \mathrm{ml}$ penicillin and streptomycin +DMEM medium), MEM.EBSS (NEAA) medium $(10 \%$ fetal bovine serum $+100 \mathrm{u} / \mathrm{ml}$ penicillin and streptomycin + MEM - EBSS +NEAA and MEM.EBSS culture medium) the base (10\% fetal bovine serum $+100 \mathrm{u} / \mathrm{ml}$ penicillin and streptomycin +MEM - EBSS medium) and RPMI.1640 medium $(10 \%$ fetal bovine serum $+100 \mathrm{u} / \mathrm{ml}$ penicillin and streptomycin +RPMI 1640 medium) in 37 . C, saturated humidity 95\%, 5\%C02 culture box in the conventional culture, subculture for subsequent experiments. (2): PBS cells in logarithmic growth phase were washed with $0.25 \%$ trypsin, about $1.0 \mathrm{ml}$, about 2 rain of the incubation medium completely digested, and then add the $2 \mathrm{ml}$ termination of digestion, the cells could completely off the wall, centrifugal. Discard the supernatant was added to culture medium of new, and counted under the microscope cell, the cell concentration was adjusted were inoculated in six larvae in L (with no anti culture fluid were cultured in 37 6C, $5 \% \mathrm{C} 02$ ), the incubator overnight. Refer to the product manual, $4099 / \mathrm{ml}, 10 \mathrm{I} . \mathrm{tg} /$ $\mathrm{ml}$, 4Ittg / $\mathrm{ml}$ siRNA fragment concentration interference in Hela cells, respectively in $2 \mathrm{H}, 24 \mathrm{~h}, 48 \mathrm{~h}], 72 \mathrm{~h}$ four time points by fluorescence microscopy to observe the transfection efficiency to determine the optimal concentration and the best transfection time. The optimal time was $48 \mathrm{~h}$ after transfection. All the interference experiments were divided into three groups: the non-transfection group, the negative transfection group and the DEC1 interference group. The $\mathrm{MCF}-7$ cells were detected as DEC1 negative control group, and each time was set with 3 holes.

(1) Hela cells in log phase, adjusted to 1 X105/ ml, were inoculated in 24 well plates, each hole 50091. Were divided into three groups: untransfected group, negative gene transfection group, FRet interference group $(n=3)$. (2) after $24 \mathrm{~h}$, the transfected group was added with the culture medium, the negative fragment transfection group and the DEC1 interference group. 12h, 24h, 48h and 96h cells were collected after transfection. (3) the cells were collected and made into cell suspension. The living cells were not stained, the dead cells were stained blue, and the number of each hole was repeated three times. (4) the following formulas: the inhibition rate of inhibition rate $=$ (control group experimental group cell total cell number) / control group total cells * 100\%. (5) Logarithmic growth cells, $0.25 \%$ trypsin into single cell suspension. (6) cells were counted and the cell concentration was adjusted to $1 \times 106 / \mathrm{ml}$. (7) 1200rpm, 4 degrees $\mathrm{C}$ centrifugal $5 \mathrm{~mm}$. (8) wash with PBS for two times, filtration, centrifugation, discard supernatant. (9) discard the supernatant, add lulPE labeled anti human FOLRI 
monoclonal antibody to each tube, incubate $30 \mathrm{rnm}$ at room temperature, gently shake the test tube every $10 \mathrm{~min}$, so as to make full use of the antibody.

\section{Results}

Comparison of cell cycle: 48h, Hela and MCF-7 cell cycle were detected by flow cytometry. The results showed that the proliferation index (PI) of Hela cells was higher than that of MCF-7 cells, and the difference was statistically significant $(\mathrm{P}<0.05)$. The percentage of MCF-7 cells in GO / G1 phase cells was higher than that in Hela cells, and the ratio of S and G2 / M cells was lower than that of Hela cells. (2) apoptosis: 48h, Hela and MCF-7 cells were detected by flow cytometry after cell passage. The results showed that MCF-7 apoptosis rate was higher than that of Hela cells, the difference was statistically significant $(\mathrm{P}<0.05)$. (1) the expression of ERK1 / mRNA gene: Hela and MCF-7 cells were collected after passage of $48 \mathrm{~h}$, and the expression level of ERK1 / 2 mRNA was detected by Real-time PCR. Hela cells ERK1 mRNA expression was obviously lower than that in MCF-7 cells, only MCF-7 cells Src mRNA relative expression was $12 \%$, the difference was statistically significant $(\mathrm{P}<0.05)$ : Hela and MCF-7 cells ERK2 mRNA relative expression showed no significant difference $(\mathrm{P}>0.05)$. See table 10. (2) the expression of ERK1 / gene protein: Hela and MCF-7 cells were collected after passage of $48 \mathrm{~h}$, the total protein was extracted, and the expression of ERKl / 2 protein was detected by Western-blot method. The results showed that Hela and MCF-7 were phosphorylated ERK1 / 2 protein expression level showed no significant difference $(\mathrm{P}>0.05)$; phosphorylation of ERK1 / 2 protein expression level was significantly lower than that of Hela cells and MCF-7 cells, the difference was statistically significant $(\mathrm{P}<0.05)$. (1) the expression of c.Jun gene mRNA: Hela and MCF-7 cells were collected after passage of $48 \mathrm{~h}$, and the expression level of c.Jun was detected by Real-time PCR. The relative expression of C - Jun in Hela cells was significantly lower than that of MCF-7 cells, which was only $7 \%$ of the relative expression of mRNA c.Jun in MCF-7 cells, and the difference was statistically significant (mRNA) $(\mathrm{P}<0.05)$. (2) the expression of c.Jun gene protein: Heh and MCF-7 cells were collected after passage of $48 \mathrm{~h}$, the total protein was extracted and the expression level of c.Jun protein was detected by Western-blot method. The results show that the phosphorylation of $\mathrm{C}$ protein expression level of Jun was higher than that of MCF-7 cells and Hela cells, the difference was statistically significant $(\mathrm{P}<0.05)$ phosphorylation in:MCF-7 cells $\mathrm{C}$ protein expression level of Jun is lower than that of Hela cells, the difference was statistically significant $(\mathrm{P}<0.05)$.

\section{Discussion}

SiRNA interference technology is the use of double stranded small RNA highly efficient, specific degradation of homologous mRNA cells in order to inhibit gene expression, this technology has been widely used in gene function research, gene therapy. In this study, DEC1 negative MCF-7 cells and DEC1 positive Hela cells 
were screened for DEC1 positive Hela cells and MCF-7 cells were used as controls. The results showed that the transfection efficiency of Hela cells was the highest at $48 \mathrm{~h}$, and the average transfection rate was about $75 \%$. The inhibition rate of DEC1mRNA by Real-time PCR showed that the inhibition rate was $47 \%$ at $48 \mathrm{~h}$. The inhibition rate of DEC 1 protein by flow cytometry showed that the inhibition rate reached $63.92 \%$ at $48 \mathrm{~h}$. However, there was no significant difference in the inhibition rate of DEC1 between the transfected cells and the control group. The results showed that the transfection effect was good. In combination with the growth of cells, the experiment was carried out to determine the $48 \mathrm{~h}$ after transfection.

ERK is an important member of the MAPK family, including ERK1 and ERK2, which can be activated by a variety of stimuli, promote cell growth and proliferation. Cytokines or growth factors through the Ras / Ra / EI water price, IEK pathway activated by phosphorylation of ERK, activation of ERK (p.ERK) can promote gene transcription regulation related genes and cell cycle and expression of a variety of cancer, leading to cell proliferation and malignant transformation. Phosphorylation of ERK is generally considered to be the activation of Ras/Raf/MAPK pathway. Most of the activated ERK were transferred to the nucleus, and a series of nuclear transcription factors C, c-Jun, Myc, C, Bcl 2 and so on, were up-regulated or down regulated by these proteins.

\section{Conclusion}

The target point increased the expression level of mRNA suggested that FRet may down regulate protein translation and modification level, resulting in decreased protein levels, while there may be a negative feedback effect, lead to the increase of the corresponding index level of mRNA. DEC1 can downregulate the expression of cervical cancer Hela cells ERK upstream factor Src mRNA and protein, suggesting that DEC1 may exist in the middle of the other factor in the activation of the ERK signalling pathway, but after transfection, upregulation of Src may be associated with the negative feedback factor by activation of ERK signalling pathway in the regulation of the specific mechanism needs further study.

\section{Acknowledgement}

The research was financially by Science Plan Project of Qiqihar City (Grant No. SFGG-201330) and Science and Technology research Program of Education Department of Heilongjiang Province (Grant No. 12531780).

\section{References}

[1] Yuan Qixia, Zhang Yifang, Fan Fengtian, Wang Kun, Selenocystine sensitizes cisplatin-induced apoptosis of human cervical cancer HeLa cells, Journal of Practical Oncology, 30(4), pp. 330-334, 2015. 
[2] Wang Min, Zheng Yan, Jia Yanfe, Li Binbin, Wang Yunshan, A Survey of a Transcriptional Regulation Factor DEC1, Shandong Science, 21(4), pp. 29-35, 2008.

[3] Zhao Xiaohong, Su Qi, Retinoid acid receptor related orphan receptor and $\alpha$ Wnt signaling pathway and tumor, International Journal of Pathology and Clinical Medicine, 31(3), pp. 234-237, 2011.

[4] Yang Chengwan, Gao Xia, Zhou Tiejun, Zhang Qiang, Effect of TGF- $\beta 1$ on epithelial-mesenchyme transitionin Hela cells of human cervical cancer, Chongqing Medicine, 44(22), pp. 3030-3033, 2015. 